\title{
Strong temperature dependencies of diblock copolymer domain spacing in a solvent mixture comprising ternary components with varied selectivity
}

\author{
Akifumi Matsushita and Shigeru Okamoto
}

The lamella formed in polystyrene-block-polyisoprene (PS- $b$-PI) swollen with solvent mixtures shows temperature-dependent domain spacing. Solvent mixtures consisting of a polystyrene (PS)-selective solvent, dimethylphthalate, and a polyisoprene (PI)-selective solvent, $n$-tetradecane, which are immiscible at temperatures below $124{ }^{\circ} \mathrm{C}$, as well as a neutral solvent, di- $n$-octylphthalate, were studied. Two molecular weights of PS- $b$-PI were used. Solutions of the lower-molecular-weight PS- $b$-PI were characterized by using small-angle X-ray scattering, whereas higher molecular weight PS- $b$-PI solutions were characterized with UV-vis spectroscopy. The domain spacings of the lamella formed in these solutions showed stronger temperature dependencies than the neat diblock copolymer or the diblock copolymer/neutral solvent system. The dependencies varied with the polymer concentration and solvent composition. The reflection peaks for a solution of the higher molecular weight PS- $b$-PI were astonishingly tunable over wavelengths between 836 and $377 \mathrm{~nm}$ in the temperature range of $20-80^{\circ} \mathrm{C}$.

Polymer Journal (2015) 47, 385-390; doi:10.1038/pj.2015.4; published online 4 March 2015

\section{INTRODUCTION}

Block copolymers (BCPs) can form microphase-separated structures by self-assembly in the molten state or in solution. Periodical structures of lamellae, gyroids, cylinders or spheres are typically formed depending on the volume fraction of constituent chains in a BCP. These structures have the potential to play important roles in nanotechnology. Many researchers have demonstrated the application of BCPs as templates for lithography, donor-accepter layers in solar cells, photonic crystals and so on. ${ }^{1-7}$ Templates are fabricated by removing one domain by using a technique such as ion etching after the microphase-separated structures form on a substrate. ${ }^{1,2}$ Large interface-to-volume ratios of microphase-separated structures can be applied to the donor-acceptor interface of solar cells to improve the efficiency. ${ }^{3,4}$ Photonic crystals are formed by the periodic allocation of two different refractive index materials and reflect certain wavelengths of light. ${ }^{5-7}$

Because the addition of selective solvents to BCPs expands the achievable morphologies, $\mathrm{BCP} /$ selective solvent systems have been studied by many researchers. ${ }^{7-17}$ Lodge and coworkers reported the phase behavior of polystyrene-block-polyisoprene (PS-b-PI) in solvents of varying selectivity. ${ }^{8}$ According to their report, highly selective solvents vary the volume fraction of domains and increase the apparent segregation power between microdomains, whereas neutral solvents decrease the segregation power without changing the volume fraction. There are many other reports ${ }^{9-15}$ that detail the behavior of
$\mathrm{BCP} /$ solvent systems, though there are few reports $7,16,17$ on $\mathrm{BCP} /$ solvent mixture systems.

In a previous study, we investigated the domain spacing dependency of lamellae formed in PS- $b$-PI with solvent mixtures at room temperature using di- $n$-octylphthalate (DOP), dimethylphthalate (DMP) and $n$-tetradecane (C14). ${ }^{7} \mathrm{DMP}$ and $\mathrm{C} 14$ are highly selective for polystyrene (PS) and polyisoprene (PI), respectively, whereas DOP is a neutral solvent for both blocks. A wide variation in the lamellar domain spacing as a function of both polymer concentration and composition of the solvent mixture was revealed.

The temperature dependency of lamellar domain spacing formed in a neat $\mathrm{BCP}$ or $\mathrm{BCP} /$ neutral solvent system (PS- $b$-PI/DOP or toluene) was studied by Hashimoto et al. ${ }^{15}$ They reported that (Equation 1) the temperature dependency of the domain spacing, $D$, as

$$
D \sim\left(\frac{1}{T}\right)^{1 / 3}
$$

where $T$ is the absolute temperature.

It can be seen that domain spacings in selective solvent systems apparently have a stronger temperature dependency than that in a neutral solvent system at temperatures sufficiently below the orderdisorder transition temperature. ${ }^{8-10}$ Our PS- $b$-PI in solvent mixtures, DOP/DMP/C14, also showed a strong temperature dependency, ${ }^{7}$ which, to the best of our knowledge, is stronger than that reported for any other system. This dependency varied with the polymer concentration and the ratio of a neutral solvent, DOP. 
In this study, with using the same PS- $b$-PI/solvent mixture systems, we examine the temperature dependency of the domain spacing of the lamellae. PS- $b$-PI with two kinds of molecular weights were studied by small-angle X-ray scattering (SAXS) and UV-vis spectroscopy. Effects of compositional ratios in solutions of PS- $b$-PI/solvent mixture upon the temperature dependency of domain spacings were thoroughly investigated with the lower-molecular-weight PS- $b$-PI. On the basis of the results, a BCP solution system whose reflection peak covers whole wavelength of visible light was developed using the higher molecular weight PS- $b$-PI. On changing the temperature from 20 to $80^{\circ} \mathrm{C}$, the latter PS- $b$-PI solution system showed a large shift in the reflectionpeak wavelength from 836 to $377 \mathrm{~nm}$, whose behavior can be applied to a tunable photonic crystal for visible light.

\section{EXPERIMENTAL PROCEDURE}

DOP, DMP, styrene, isoprene, toluene, sodium, methanol and dichloromethane were purchased from Nacalai Tesque Inc. (Kyoto, Japan). $n$-tetradecane (C14), sec-butyllithium in cyclohexane/n-hexane and tert-butylmagnesium chloride in tetrahydrofuran were obtained from Kanto Chemical Co., Inc. (Tokyo, Japan).

Symmetric diblock copolymers of PS- $b$-PI (SIs) were synthesized by living anionic polymerization under high vacuum, as described elsewhere. ${ }^{7}$ The molecular weights of Sls were evaluated by gel permeation chromatography using a Tosoh GPC system (Tosoh, Tokyo, Japan) equipped with TSK gel columns (GMH, G4000H, G2000H and $\mathrm{G} 1000 \mathrm{H}$ ) and a refractive index detector (ERC 7522: Erma Inc., Tokyo, Japan). Tetrahydrofuran was used as an eluent and PS samples were used as molecular weight standards. The compositions of Sls were determined by ${ }^{1} \mathrm{H}$ NMR in $\mathrm{CDCl}_{3}$ with a Bruker 200-MHz spectrometer (Avance 200: Bruker, Billerica, MA, USA). The PI blocks have $90.6 \pm 2 \% 1,4$-isoprene units as determined by ${ }^{1} \mathrm{H}$ NMR. The volume fractions of PS in Sls were estimated from the known densities of the respective blocks $\left(1.05 \mathrm{~g} \mathrm{~cm}^{-3}\right.$ for PS and $0.92 \mathrm{~g} \mathrm{~cm}^{-3}$ for $\left.\mathrm{PI}^{18}\right)$. The characteristics are summarized in Table 1 . The specimens are denoted by SI$\mathrm{X}$, where $\mathrm{X}$ is the total molar mass of the polymer in $\mathrm{kDa}$.

To assist the systems in reaching equilibrium, all solutions of Sls in the three solvents were prepared using dichloromethane as a cosolvent. The cosolvent was removed by drying in air at $60^{\circ} \mathrm{C}$ until a constant weight was achieved.

Glass tubes with a $3.4 \mathrm{~mm}$ inner diameter and $5.0 \mathrm{~mm}$ outer diameter were used as sample cells for UV-vis measurements. Reflectivity spectra measurements were conducted with a UV-vis spectrophotometer (Ocean Optics DH-2000-BAL (Ocean Optics, Dunedin, FL, USA) and Maya-2000 PRO (Ocean Optics) as a light source and detector, respectively) over the wavelength range from 200 to $1100 \mathrm{~nm}$. Normal reflection spectra were measured with the incident light perpendicular to the surface of samples. The optical fiber from the light source and the one to the detector are combined in the detector probe; hence, the incident light is also emitted from the probe. Thus, the detector collect vertically reflected lights from lattice planes oriented parallel to the surface of a cell. The temperature was controlled with a Box Cool $(\mathrm{Ohm}$ Electric Inc., Tokyo, Japan).

Temperature sweep in the SAXS measurements was performed from 50 to $180^{\circ} \mathrm{C}$ in a heating cycle on the beamlines $40 \mathrm{~B} 2$ and $08 \mathrm{~B} 2$ at SPring 8 and $8 \mathrm{~S} 3$ at AichiSR, Japan. Temperature was controlled with a TMS 94 controller (Linkam Scientific Instruments Ltd, Surrey, UK). The X-ray wavelength was $0.15 \mathrm{~nm}$. An imaging plate (R-AXIS VII, Rigaku Corporation (Akishima, Japan) for 40B2 and R-AXIS IV++, Rigaku Corporation for $08 \mathrm{~B} 2$ and $8 \mathrm{~S} 3$ ) was used as a

Table 1 Characteristics of Sls used in this study

\begin{tabular}{lccl}
\hline Polymer code & $M_{w} \times 10^{-3}$ & $M_{w} / M_{n}$ & $\phi P S^{\mathrm{a}}$ \\
\hline SI-117 & 117 & 1.05 & 0.48 \\
SI-655 & 655 & 1.09 & 0.38
\end{tabular}

Abbreviations: PS, polystyrene; SIs, PS- $b$-PI diblock copolymers.

${ }^{a} \phi_{P S}$ is the volume fraction of a PS block. detector. The beam sizes at the detector positions were $0.2 \times 0.2,0.15(\mathrm{H}) \times 0.25$ $(\mathrm{V}), 0.67(\mathrm{H}) \times 0.14(\mathrm{~V}) \mathrm{mm}^{2}$ for $40 \mathrm{~B} 2,08 \mathrm{~B} 2$ at SPring8 and $8 \mathrm{~S} 3$ at AichiSR, respectively. Additional SAXS measurements were carried out on a Nanoviewer (Rigaku Corporation) instrument. The X-ray beam of the $\mathrm{CuK} \alpha$ line $(\lambda=0.154$ $\mathrm{nm}$ ) was incident on samples. The beam size at the detector position was 0.63 $(\mathrm{H}) \times 0.63(\mathrm{~V}) \mathrm{mm}^{2}$. Scattering intensity was measured with an imaging plate (R-AXIS IV++, Rigaku). A cell of thickness $0.98 \mathrm{~mm}$ with windows made of Kapton (Du Pont-Toray Co., Ltd., Tokyo, Japan) was used for these SAXS measurements. The scattering intensity was corrected for absorption of samples, air scattering from an empty cell (also two pieces of thin Kapton window) and dark current, that is, electronic noise of the detector.

\section{RESULTS AND DISCUSSION}

Several solutions of SI-117 were prepared in the ternary solvent mixtures, DOP/DMP/C14. The volume ratio of the selective solvents, DMP:C14, was fixed at 51:49.

Figure 1 shows temperature dependency of SAXS, measured in a heating cycle, from solutions at the SI-117 concentration of $30 \mathrm{vol} . \%$ with 10 vol.\% of the neutral solvent, DOP, in the DOP/DMP/C14 solvent mixture. Here, $q$ is the magnitude of the scattering vector defined by $q=4 \pi \sin \theta / \lambda$, where $2 \theta$ and $\lambda$ are the scattering angle and the wavelength, respectively. The value of $q$ is reciprocally proportional to a domain spacing, $D$, that is, $q=2 \pi / D$. These profiles exhibited multiple scattering peaks. The peak position ratios relative to the primary peak are at the integer multiples of the primary peak position, indicating that lamellar structures are formed at all of these three different temperatures. Though not shown here, lamellar structures were also formed in all the solutions prepared (20, 30 and $40 \mathrm{vol} . \%$ SI-117 with $10,0-50$ and $0-30$ vol.\% of DOP in the DOP/DMP/C14 solvent mixture, respectively) over the whole temperature region studied $\left(50-180^{\circ} \mathrm{C}\right)$. Note that the 20 vol.\% SI-117 solution without DOP macroscopically phase-separated at lower temperatures (50 and $60^{\circ} \mathrm{C}$ ).

The positions of the scattering peaks shifted toward the larger $q$-values as the temperature increased, that is, the domain spacing

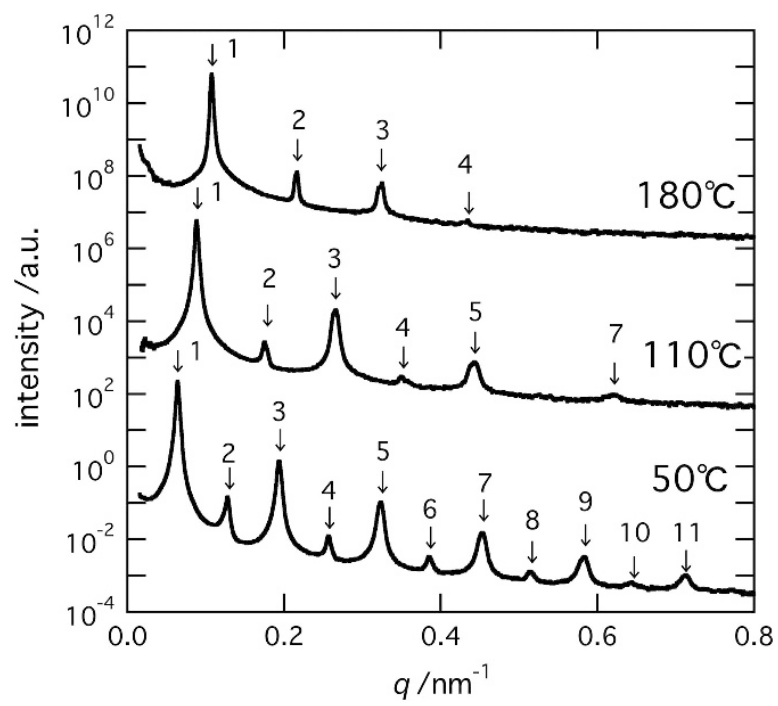

Figure 1 Strong temperature dependencies of diblock copolymer domain spacing in a solvent mixture comprising ternary components with varied selectivity. SAXS profiles of 30 vol.\% SI-117 with 10 vol.\% DOP in the DOP/ $\mathrm{DMP} / \mathrm{C} 14$ solvent mixture at 50,110 and $180^{\circ} \mathrm{C}$. The profiles are vertically shifted to avoid overlaps. The peak positions relative to the first-order peak are indicated. 
decreased. This basically arose from the fact that the segregation power between two block chains of a neat BCP decreases roughly proportionally to the reciprocal of an absolute temperature, $T$. Here, segregation power is generally expressed by $\chi N$, where $\chi$ and $N$ are the Flory-Huggins interaction parameter between monomeric units of blocks and the degree of polymerization, respectively. Note that, in this study, we used a $\mathrm{BCP} /$ solvents blend system; thus, we have to take into account all the possible combinations of the constituent molecules, that is, the effective segregation power $\chi_{\text {eff }} N$ instead of $\chi N$ should be taken account of. ${ }^{8}$ Further detail on the temperature dependence of the domain spacings will be discussed later.

In the SAXS observed at 50 and $110^{\circ} \mathrm{C}$, it can also be seen that the intensity of the even-numbered peaks are relatively weak or missing. This extinction is attributed to the fact that the form factors of the lamellae become close to zero (destructive interference) at the corresponding $q$-values because the PS and PI domains have almost identical volume fractions. ${ }^{19}$ However, this extinction is not obvious at $180^{\circ} \mathrm{C}$, indicating that the volume fraction has become asymmetric. Indeed, the volume fraction of the PS phase was $\sim 51 \pm 1$ vol.\% at 50 and $110^{\circ} \mathrm{C}$, whereas it increased up to $\sim 53 \pm 3$ vol. $\%$ at $180^{\circ} \mathrm{C}$. These volume fractions were estimated by fitting the profiles with theoretical scattering curves. Note that, as we discussed in previous report, DOP has higher affinity to DMP than C14. ${ }^{7}$ Hence we considered the solvent mixture swell PS domain more than PI domain by slightly larger distribution of DOP, as will be discussed later. Speculatively, this slight asymmetry may be attributed to a change in the spatial distributions of the solvents with the decrease in selectivity as discussed later or the thermal expansion coefficients of the constituents.

Now, we discuss the SAXS results on 30 vol.\% SI-117 solutions with various ratios of DOP in the solvent mixture, DOP/DMP/C14. D, evaluated from a primary peak position, is plotted against a reciprocal absolute temperature in a double logarithmic scale in Figure 2a. Measurements were conducted in a heating cycle with the stepwise increment of $10^{\circ} \mathrm{C}$ from 50 to $180^{\circ} \mathrm{C}$ with $10 \mathrm{~min}$ of annealing at each temperature before a measurement. There are two distinct features in Figure 2a; $D$ markedly increases with decreasing temperature; $D$ also increases and the slope becomes bigger as the DOP content decreases. The value of $D$ in the solution without DOP surprisingly increased from 62 to $115 \mathrm{~nm}$. It is also clearly seen that the slope is bigger in the lower temperature region and the curvatures of the graphs resemble each other. Thus, the graphs are horizontally shifted along the axis of $\log \left(T^{-1}\right)$ to obtain a master curve with the reference of the DOP concentration of 0 vol. $\%$ as shown in Figure $2 \mathrm{~b}$. The logarithmical shit factors, $\log a_{\mathrm{T}}$, calculated as mentioned later are $-0.0236,-0.0936$ and -0.159 for the DOP concentration of 10,30 and $50 \mathrm{vol} . \%$, respectively. In the lower temperature region, $T \leqslant 120^{\circ} \mathrm{C}$, that is, $\log$ $\left(T^{-1}\right) \geqslant-2.595$ in Figure $2 \mathrm{~b}, \mathrm{D}$ exhibits approximately linear large increase, whereas the slope becomes smaller at the higher temperature and reaches around $1 / 3$, which is the slope reported for a neat BCP or a BCP in a neutral solvent.

As we reported in our previous study, ${ }^{7}$ the domain spacing decreases with increasing DOP content because a segregation power is shielded by a neutral solvent, that is, $\chi_{\text {eff }}$ decreases at higher DOP concentrations. In addition, at higher DOP concentrations, the slope is close to the value reported for a neat $\mathrm{BCP}$ or a $\mathrm{BCP} /$ neutral solvent system, ${ }^{15}$ that is, that in $D \sim(1 / T)^{1 / 3}$ because the solvent mixture becomes virtually neutral. Similarly, it is considered that at higher temperatures in Figure $2 \mathrm{~b}$, the slope is smaller as mentioned above because the selectivity of the solvent mixture decreases. However, the slope of the master curve in the lower temperature region, $50^{\circ} \mathrm{C} \leqslant T$
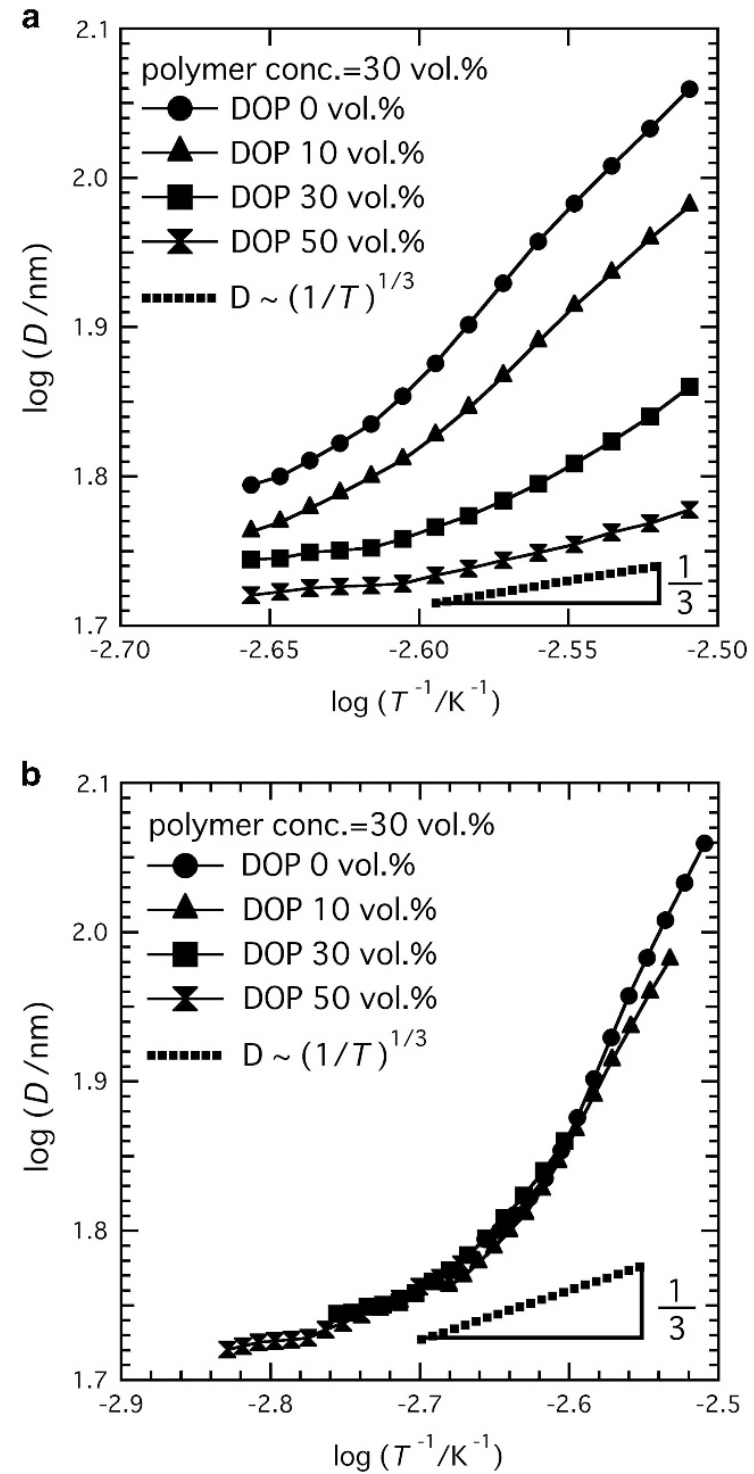

Figure 2 (a) Temperature dependencies of the domain spacing in 30 vol.\% SI-117 solutions with 0, 10, 30 and 50 vol.\% DOP. (b) A master curve obtained by horizontally shifting the graphs at the DOP concentrations of 10 , 30 and 50 vol.\% with the logarithmical shift factors of $-0.0236,-0.0936$ and -0.159 , respectively.

$<120^{\circ}$, that is, $-2.595 \leqslant \log \left(T^{-1}\right) \leqslant-2.509$, is significantly larger than $D \sim(1 / T)^{1 / 3}$. The value of $\alpha$ for $D \sim(1 / T)^{\alpha}$ is $\sim 2.2$ and 1.8 for the solutions at the DOP concentrations of 0 and 10 vol.\%, respectively. The large values of the slope indicate that $\chi_{\text {eff }}$ varies greatly with temperature change because the miscibility of DMP and C14 strongly depends on temperature, which is demonstrated later. The selective solvents initially locating largely in their respective preferential domains, the increase in the miscibility between them leads to migrations of the solvents into the opposite domains; as a result, $\chi_{\text {eff }}$ decreases.

The temperature dependence of the miscibility change between DMP and C14 is the key to understand the astonishing increase of $D$ and the change in the slope of the master curve with temperature. Thus, we prepared the mixture of DMP and C14 in a vial at the volume fraction ratio of 51:49 that is the same as that of the mixture for the polymer solutions. This mixture separated into two phases, 
whereas solvent mixtures containing DOP at the concentration of more than 34 vol.\% was in one phase at room temperature $\left(26^{\circ} \mathrm{C}\right)$. Therefore, we prepared solvent mixtures at the DOP concentration less than 34 vol.\%, in fact, at 0,10 and 30 vol.\%. These solutions were in the two-phase state: the DMP-rich phase with DOP/slight amount of C14, and the C14-rich phase with DOP/slight amount of DMP. These solutions were cooled down from the high temperature $20^{\circ} \mathrm{C}$ above the critical solution temperatures $\left(T_{\mathrm{c}}\right)$ obtained below, at which they are in one-phase state, gradually to the low temperature at which they go into two-phase state in order to locate their critical solution temperatures. The critical solution temperatures thus obtained were 124,103 and $47^{\circ} \mathrm{C}$, that is, $\log \left(T^{-1}\right)=-2.599,-2.575$ and -2.505 , for the DOP concentrations of 0,10 and $30 \mathrm{vol} . \%$, respectively. As a comparison, the theoretical order-disorder transition temperature of SI-117 was estimated to be $822^{\circ} \mathrm{C},{ }^{20}$ which is much higher than the critical solution temperatures of the mixtures, DMP/C14/DOP. In other words, the fact that their critical solution temperatures of the solvent mixtures were much lower than the order-disorder transition temperature of neat SI-117 and the fact that the segregation power between DMP and C14 was so strong as to enhance the segregation between PS and PI at the temperature below $T_{\mathrm{c}}$ indicate that the temperature dependence of $\chi$ between DMP and C14 is much stronger than that between the block chains. Note that although the data not shown here, the volume fractions of PS for these solutions were constant $(51 \pm 2$ vol. $\%)$ below $130{ }^{\circ} \mathrm{C}$ except the solution at the DOP concentration of $50 \mathrm{vol} . \%$ whose SAXS profile obtained with the inhouse instrument (Nanoviewer) had very weak higher order peaks and thus the volume fraction could not be evaluated. Similarly, the volume fractions of PS at $180{ }^{\circ} \mathrm{C}$ were obtained only for the solutions at the polymer concentrations of 30 and 40 vol.\% and the DOP concentration of 10 vol.\%; the PS fractions were $53 \pm 3$ vol.\%. Therefore, it may be safely expected that the volume fractions were nearly constant over the experimental temperature range. Hence, it is conceivable the compositional ratio of DMP/C14/DOP in each domain was also nearly constant for all of the solutions and the temperature dependence of $\chi$ between DMP and C14 makes the domain spacing so big and change so much as shown in Figure 2. The shift factors mentioned above were so calculated as to match the critical temperatures with the reference temperature of $124^{\circ} \mathrm{C}$ ( $T_{\mathrm{c}}$ for the DOP concentration of 0 vol.\%), that is, the shift factor is defined by $a_{T}=\log \left\{\left(273.15+T_{\mathrm{c}}\right) /(273.15+124)\right\}$. Note that the critical temperature was not obtained for the solution at the DOP concentration of 50 vol.\%; thus, the graph for this solution was horizontally shifted as it well overlaps the master curve already constructed with the other graphs; the critical temperature $\left(\sim 2.5^{\circ} \mathrm{C}\right)$ estimated by the shift factor was much below the ambient temperature. It is also worth while to note that the solvent mixtures at the DOP concentration of 0 and $10 \mathrm{vol} . \%$ at the lower temperature, log $\left(T^{-1}\right) \geqslant-2.572$, were so strongly segregated that they increased the segregation power between the block chains as rationally concluded by Figure 3 shown below. This means that $\chi$ between DMP and C14 at room temperature is much higher than $\chi N$ of neat SI-117. Note also that the segregation power between DMP and C14 in the solution at the low polymer concentration of $20 \mathrm{vol} . \%$ without DOP was too strong. Therefore, the solution macroscopically phase-separated at lower temperatures $\left(50\right.$ and $\left.60^{\circ} \mathrm{C}\right)$ as mentioned above. In other words, PS- $b$-PI behaves as a compatibilizing agent between DMP and C14.

SAXS measurements were conducted on various SI-117 solutions at the DOP concentration of $10 \mathrm{vol} . \%$ as well as a neat SI- 117 to elucidate the temperature dependence of the domain spacings for various polymer concentrations. The SAXS profiles, though not shown

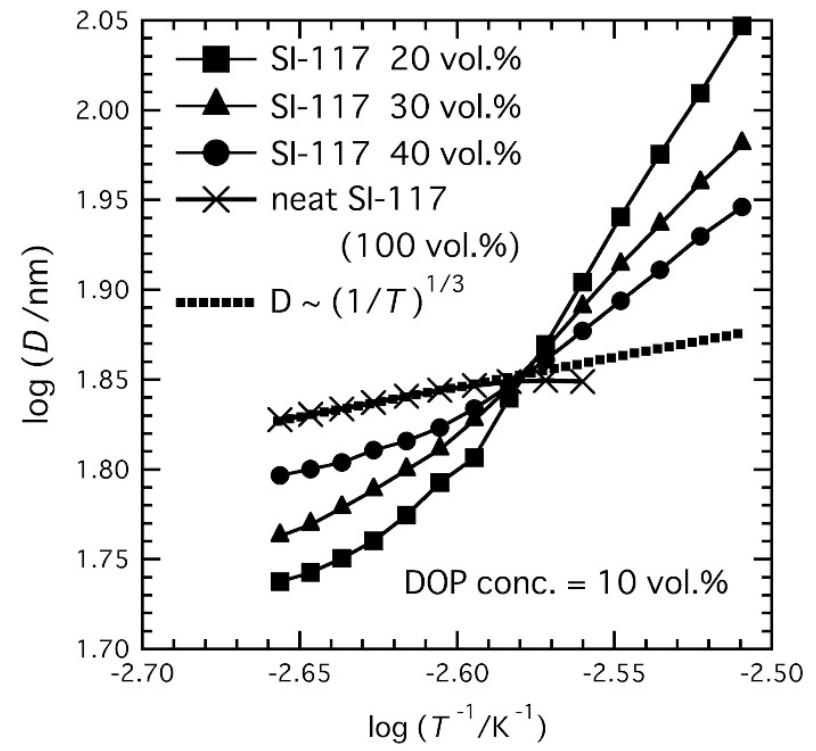

Figure 3 Temperature dependencies of the domain spacing in 20, 30 and 40 vol.\% SI-117 solutions with 10 vol.\% DOP.

here, exhibited lamellar structures as mentioned above whose peak intensity ratios suggested that all of the systems had almost symmetric volume fractions between the phases. The domain spacings are shown in Figure 3. The domain spacing in the neat SI-117 followed $D \sim(1 / T)^{1 / 3}$ between 120 and $180^{\circ} \mathrm{C}$, that is, $-2.656 \leqslant \log$ $\left(T^{-1}\right) \leqslant-2.595$, whereas below $110^{\circ} \mathrm{C}$, that is, $\log \left(T^{-1}\right) \geqslant-2.583$, the slope deviates from $D \sim(1 / T)^{1 / 3}$. It is attributed to that SI- 117 does not reach an equilibrium state because polystyrene has a glass transition temperature $\sim 100^{\circ} \mathrm{C}$. In the higher temperature region, $110^{\circ} \mathrm{C} \leqslant T \leqslant 180^{\circ} \mathrm{C}$, that is, $-2.656 \leqslant \log \left(T^{-1}\right) \leqslant-2.583$, the domain spacings of the solutions increased with the increase in the polymer concentration. The asymptotic behavior is close to $D \sim(1 / T)^{1 / 3}$. In contrast, in the lower temperature region, $100{ }^{\circ} \mathrm{C} \leqslant T \leqslant 50^{\circ} \mathrm{C}$, that is, $-2.572 \leqslant \log \left(T^{-1}\right) \leqslant-2.509$, the slopes for the solutions show greater deviation from $D \sim(1 / T)^{1 / 3}$ as the polymer concentration decreases. The largest value of $\alpha$ was obtained for the $20 \mathrm{vol} . \%$ polymer solution between 50 and $100^{\circ} \mathrm{C}$, that is, $-2.572 \leqslant \log \left(T^{-1}\right) \leqslant-2.509$; the slope was 2.9.

As mentioned above, at higher/lower temperatures, the solutions with higher/lower polymer concentrations have larger domain spacings, respectively. These opposite tendencies below and above $\sim 105^{\circ} \mathrm{C}\left(\log \left(T^{-1}\right) \lessgtr-2.578\right)$ indicate a reversal of the dependency of $\chi_{\text {eff }}$ on the polymer concentration. The addition of the solvent mixture at a temperature below the critical solution temperature $\left(103^{\circ} \mathrm{C}\right)$, that is, $\log \left(T^{-1}\right)<-2.575$ increases $\chi_{\mathrm{eff}}$; in other words, the decrease in the polymer concentration increases the domain spacing because the phase-separated solvent mixture has a stronger segregation power than that between the block chains and thus works as a strongly selective solvent. By contrast, the molecularly mixed solvent at a higher temperature above $103^{\circ} \mathrm{C}$, working as a neutral solvent, shields the segregation power between the block chains, that is, leads to the decrease in $\chi_{\text {eff. }}$ More interestingly, all the solutions and neat SI-117 have the same domain spacings at $\sim 105^{\circ} \mathrm{C}$, where the abovementioned opposite tendencies balanced for all of the polymer concentrations. Here, it is worth to note that the structures were thermo-reversibly reproducible. As a typical example, temperature dependency of the SAXS profile from the solution at the polymer 
concentration of $40 \mathrm{vol} . \%$ and the DOP concentration of $10 \mathrm{vol} . \%$ is shown in Supplementary Figure S1. The change in the peak position of the primary peak, that is, the change of the domain spacing finished within the first $10 \mathrm{~min}$ after the temperature jump, indicating that the structure was equilibrated within $10 \mathrm{~min}$ as shown. Further more, the profiles before and after annealing at a higher temperature were in good agreement with each other.

Figure 4 shows the reflectance spectra of a SI-655 solution (SI-655/ DOP/DMP/C14 $=6.8 / 27.0 / 26.0 / 40.2($ vol. $\%)$ ) from 20 to $80^{\circ} \mathrm{C}$. It is worthy to note that this solution has less amount of DMP than in the SI-117 solutions because the volume fractions of the PS domains in the latter were slightly larger than $50 \mathrm{vol} . \%$ as mentioned above, which is presumably because slightly larger amount of DOP located in the PS domain because of the affinity between DMP and DOP. SAXS measurements conducted on this solution confirmed that lamella structures were formed in the temperature region from 20 to $80^{\circ} \mathrm{C}$ and the volume fractions of PS and PI were close to 50 vol.\% as revealed by well-suppressed even-numbered peaks (Supplementary Figure S2). The domain spacings of this solution are large enough to reflect visible light, whereas the domain spacings of the SI-117 solutions are not. The reflectance spectra measured at temperatures between 20 an $80^{\circ} \mathrm{C}$ are shown in Figure 4. However, there were higher order reflection peaks, whose peak position relative to the primary peak position was $1 / \sqrt{ } 2$ (Supplementary Figure S3), indicating that the structure is spherical microdomains on a body-centered cubic lattice. Hence, it is conceivable that the structure in equilibrium is lamellae (inside the solution) but only the structure on the surface of the glass container is transformed to BCC. DOP and DMP presumably have affinity for glass because they have polar groups (carbonyl) in their structures and hence accumulated near the surface of the glass container. We consider the accumulation transformed the structure to BCC. However, the relative shifts of the peaks observed in SAXS and reflectance spectra were in good accordance. Actually, the peak shifts were observed from 0.0469 to $0.0215 \mathrm{~nm}^{-1}$ (Supplementary Figure S2) and from 377 to $836 \mathrm{~nm}$ by SAXS and spectroscopy, respectively. The shifts in the domain spacings estimated from the above results using $2 \pi / \mathrm{q}$ and Equation (2) were from 134 to $292 \mathrm{~nm}$ and from 128 to 283 , respectively. Therefore, we investigated the temperature response by reflectance spectroscopy instead of SAXS. Note that a measurement by reflectance spectroscopy is quicker than

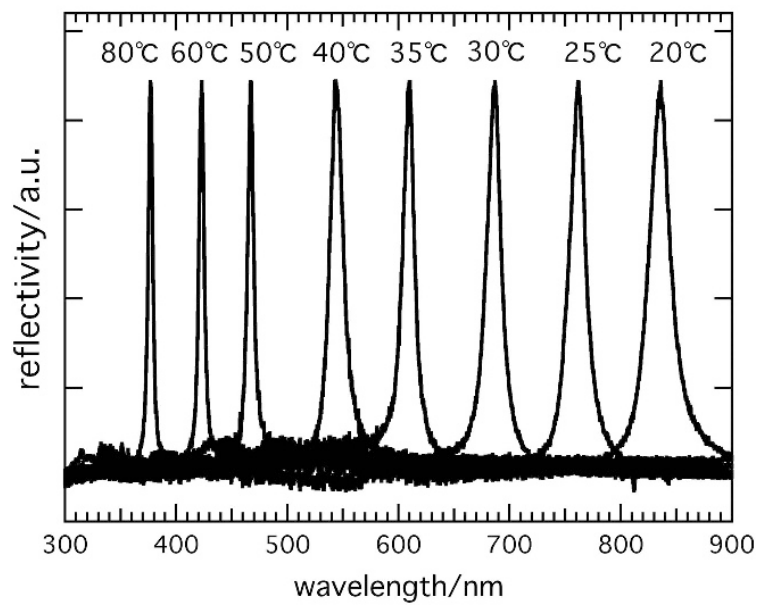

Figure 4 Temperature dependencies of the reflectance spectra of a SI-655 solution (SI-655/DOP/DMP/C14 =6.8/27.0/26.0/40.2 (vol.\%)). by SAXS. In Figure 4, the wavelength of the reflection peak shifted from 836 to $377 \mathrm{~nm}$ over this temperature range. The following equation shows the relationship between the wavelength of the first reflection peak $\left(\lambda_{\text {peak }}\right)$ and the domain spacing $(D)$ :

$$
\lambda_{\text {peak }}=2 n D
$$

where $n$ is the average refractive index of the reflecting elements. The average refractive index for SI-655 calculated from the refractive indices of the constituent components (1.59 for PS, 1.521 for PI, ${ }^{18}$ 1.515 for DMP, 1.485 for DOP and 1.429 for C14; the catalog values from the suppliers) and their volume fractions is 1.475 . Therefore, the domain spacings calculated from Equation (2) for this solution at 20 and $80^{\circ} \mathrm{C}$ are 283 and $128 \mathrm{~nm}$, respectively. The $\alpha$ value of the slope between 20 and $50^{\circ} \mathrm{C}$ obtained from a plot of $D$ vs $(1 / T)$ for SI-655 is 6.0 though not shown here. This result suggests that the SI-655 solution has such a larger shift in a domain spacing with temperature than SI-117 solutions because of the following reasons: the range of the effective segregation power $\left(\chi_{\text {eff }} N\right)$ was expanded by the higher molecular weight, that is, by the large value of $N$; the larger range was also achieved by the lower polymer concentration as easily expected by Figure 3. Note that macrophase separation was successfully avoided by the large amount of DOP although the polymer concentration was so low.

Finally, we investigated the temperature responses to a temperature jump (T-jump) and a temperature sweep (T-sweep). We prepared two sets of heat enclosures for a temperature jump: one at $30^{\circ} \mathrm{C}$ and the others at 20,40 or $70^{\circ} \mathrm{C}$. Temperature jump from 20,40 or 70 to $30^{\circ} \mathrm{C}$ was performed by moving a sample cell quickly from one heat enclosure to another. Note that $T_{\mathrm{c}}$ for this solution is $47^{\circ} \mathrm{C}$. Temperature of a cell after a T-jump was equilibrated within $\sim 30$ and $\sim 45 \mathrm{~s}$ after the jump from 20 and $40^{\circ} \mathrm{C}$ and from $70^{\circ} \mathrm{C}$, respectively. Domain spacings reached the equilibrated value at $30^{\circ} \mathrm{C}$ within the discrepancy of $1 \%$ in the same time as the temperature did so after the T-jumps as shown by the shifts of the reflection peaks (Supplementary Figure S4). This indicates that the structural change is as quick as the temperature change. Next, temperature was increased/ decreased at the average rates of $\sim+0.088 /-0.118^{\circ} \mathrm{C} / \mathrm{min}$ between 20 and $70^{\circ} \mathrm{C}$. The peak positions were in good accordance with the peak position of the equilibrated structures in Figure 4 during the whole T-sweeps as shown in Supplementary Figure S5.

\section{CONCLUSION}

We showed the temperature dependence of the domain spacing of the lamella structures formed in PS- $b$-PI solutions prepared in the solvent mixtures of DMP, DOP and C14. DMP and C14 are highly selective for polystyrene and PI, respectively, whereas DOP is a neutral solvent for both of the blocks. DMP and $\mathrm{C} 14$ are immiscible $\left(\leqslant 124^{\circ} \mathrm{C}\right)$, but DOP is a mutual solvent for all the components. Solutions of the lower-molecular-weight SI-117 were studied by SAXS in the temperature region from 50 to $180^{\circ} \mathrm{C}$ to elucidate the effect of the polymer concentration and solvent composition on the temperature dependence of the domain spacing. DMP and C14 selectively distribute in the polystyrene and PI domains, respectively, and sustain lamella structure. The strong temperature dependence of the segregation power between the selective solvents enhanced the temperature dependence of the domain spacing. The degree of the enhancement was well controlled by the addition of DOP. In all of the solutions, lamellar structures were also formed irrespective of the concentrations of the polymer or the solvents. They also showed a strong temperature dependency of the domain spacings below the critical solution temperatures of the solvent mixtures of DOP/DMP/C14, whereas 
they had a much weaker temperature dependency above the critical temperatures, which is closer to that of a neat block copolymer or a block copolymer in a neutral solvent. The solution prepared with the higher molecular weight SI-655, characterized by UV-vis spectrometry, reflected the whole visible light whose wavelength ranges from 836 to $377 \mathrm{~nm}$ with the increase in temperature from 20 to $80^{\circ} \mathrm{C}$. The structures formed in the solutions exhibited thermo-reversible reproducibility and quick temperature response. This temperatureresponsible color change of reflected light in the mild-temperature region has a great potential for future thermochromic applications including photonic crystals.

\section{ACKNOWLEDGEMENTS}

The synchrotron radiation experiments were performed at BL40B2 and BL08B2 of SPring-8 with the approval of the Japan Synchrotron Radiation Research Institute (JASRI) (Proposal Numbers 2011B1502, 2012B1476, 2013B1689 and 2014A1423), and at BL8S3 of Aichi Synchrotron Radiation Center, Aichi Science \& Technology Foundation, Aichi, Japan (Proposal Numbers 201402054 and 201405071). This work was supported by JSPS KAKENHI Grant Numbers 21550208 and 24550250.

1 Sundrani, D., Darling, S. B. \& Sibener, S. J. Hierarchical assembly and compliance of aligned nanoscale polymer cylinders in confinement. Langmuir 20 5091-5099 (2004).

2 Cheng, J. Y., Rettner, C. T., Sanders, D. P., Kim, H.-C. \& Hinsberg, W. D. Dense selfassembly on sparse chemical patterns: Rectifying and multiplying lithographic patterns using block copolymers. Adv. Mater. 20, 3155-3158 (2008).

3 Botiz, I. \& Darling, S. B. Self-assembly of poly(3-hexylthiophene)-block-polylactide block copolymer and subsequent incorporation of electron acceptor material. Macromolecules 42, 8211-8217 (2009).

4 Sun, S.-S. Design of a block copolymer solar cell. Sol. Energy Mater. Sol. Cells 79, 257-264 (2003)

5 Paquet, C. \& Kumacheva, E. Nanostructured polymers for photonics. Mater. Today 11 , 48-56 (2008).
6 Yoon, J., Lee, W. \& Thomas, E. L. Thermochromic block copolymer photonic gel Macromolecules 41, 4582-4584 (2008).

7 Matsushita, A. \& Okamoto, S. Tunable photonic crystals: Control of the domain spacings in lamellar-forming diblock copolymers by swelling with immiscible selective solve nts and a neutral solvent. Macromolecules 47, 7169-7177 (2014).

8 Hanley, K. J., Lodge, T. P. \& Huang, C.-I. Phase behavior of a block copolymer in solvents of varying selectivity. Macromolecules 33, 5918-5931 (2000).

9 Lai, C., Russel, W. B. \& Register, R. A. Scaling of domain spacing in concentrated solutions of block copolymers in selective solvents. Macromolecules 35 4044-4049 (2002).

10 Valkama, S., Kosonen, H., Ruokolainen, J., Haatainen, T., Torkkeli, M., Serimaa, R., Brinke, G. T. \& Ikkala, O. Self-assembled polymeric solid films with temperatureinduced large and reversible photonic-bandgap switching. Nat. Mater. 3, 872-876 (2004).

11 Chastek, T. Q. \& Lodge, T. P. Measurement of gyroid single grain growth rates in block copolymer solutions. Macromolecules 36, 7672-7680 (2003).

12 Watanabe, H., Kanaya, T. \& Takahashi, Y. Equilibrium elasticity of diblock copolymer micellar lattice. Macromolecules 34, 662-665 (2001).

13 Jang, Y., Chung, J., Lee, S., Lim, H., Baek, H. \& Kang, Y. Block copolymer photonic mesogels exhibiting dual volume phase transitions. Polym. Bull. 70, 593-601 (2013).

14 Suo, T., Yan, D., Yang, S. \& Shi, A.-C. A theoretical study of phase behaviors for diblock copolymers in selective solvents. Macromolecules 42, 6791-6798 (2009).

15 Hashimoto, T., Shibayama, M. \& Kawai, H. Ordered structure in block polymer solutions. 4. Scaling rules on size of fluctuations with block molecular weight, concentration, and temperature in segregation and homogeneous regimes. Macromolecules 16, 1093-1101 (1983).

16 Alexandridis, P., Olsson, U. \& Lindman, B. A record nine different phases (four cubic, two hexagonal, and one lamellar lyotropic liquid crystalline and two micellar solutions) in a ternary isothermal system of an amphiphilic block copolymer and selective solvents (water and oil). Langmuir 14, 2627-2638 (1998).

17 Ando, K., Yamanaka, T., Okamoto, S., Sakamoto, N., Yamaguchi, D., Koizumi, S. Hasegawa, H. \& Koshikawa, N. SCFT simulation and SANS study on spatial distribution of solvents in microphase separation induced by a differentiating non-solvent in a semidilute solution of an ultra-high-molecular-weight block copolymer. J. Phys.: Conf. Ser. 247, 012040 (2010).

18 Brandrup, J., Immergut, E. H., Grulke, E. A., Abe, A. \& Bloch, D. R. Polymer Handbook (John Wiley and Sons, New York, 1999).

19 Ogawa, T., Sakamoto, N., Hashimoto, T., Han, C. D. \& Baek, D. M. Effect of volume fraction on the order-disorder transition in low molecular weight polystyrene-block-polyisoprene copolymers. 2. Order-disorder transition temperature determined by small-angle X-ray scattering. Macromolecules 29, 2113-2123 (1996).

20 Lodge, T. P., Pan, C., Jin, X., Liu, Z., Zhao, J., Maurer, W. W. \& Bates, F. S. Failure of the dilution approximation in block copolymer solutions. J. Polym. Sci. B: Polym. Phys. 33, 2289-2293 (1995)

Supplementary Information accompanies the paper on Polymer Journal website (http://www.nature.com/pj) 